\title{
Existence of the solution of fractional integral inclusion with time delay
}

\author{
Rabha W. Ibrahim and Hamid A. Jalab
}




\title{
EXISTENCE OF THE SOLUTION OF FRACTIONAL INTEGRAL INCLUSION WITH TIME DELAY
}

\author{
RABHA W. IBRAHIM AND HAMID A. JALAB
}

Received 30 April, 2009

\begin{abstract}
In this article, we establish the existence of solutions for integral inclusion of fractional order. The study holds in the case when the set-valued (multi-valued) function has convex and non-convex values. Also we illustrate our results with examples to which our abstract theory applies.
\end{abstract}

2000 Mathematics Subject Classification: 34G10, 26A33, 34A12, 42B05

Keywords: fractional calculus, set-valued function, integral inclusion

\section{INTRODUCTION}

The class of fractional operator equations of various types plays very important role not only in mathematics but also in physics, control systems, dynamical systems and engineering. Naturally, such equations required to be solved. There are numerous books focused in this direction, that is concerning the linear and nonlinear problems involving different types of fractional derivatives as well as integral see [20,22,23, 27, 28]. In [13, 14, 17], El-Sayed and A. G. Ibrahim gave the concept of the definite integral of fractional order for set-valued function. Integral and integro-differential (linear and nonlinear) in abstract spaces has been studied by several authors see [3, 18,24,25]. Applications of this type of problem arise in the study of control systems (see $[1,2,10])$. This paper concerned with the following fractional integral inclusion.

$$
u(t)-\sum_{i=1}^{n} b_{i}(t) u\left(t-\tau_{i}\right) \in I^{\alpha} F(t, u(t)) ; 0<\alpha \leq 1, t \in J:=[0, T],
$$

where $\tau_{i} \leq t \in J, \forall i=1, \ldots, n, F: J \times \mathbb{R} \rightarrow \mathcal{P}(\mathbb{R})$ is a set-valued function with nonempty values in $\mathbb{R}, \mathcal{P}(\mathbb{R})$ is the family of all nonempty subsets of $\mathbb{R}, I^{\alpha} F(t, u(t))$ are the definite integral for the set-valued functions $F$ of order $\alpha$ which is defined as

$$
I^{\alpha} F(t, u(t))=\left\{\frac{1}{\Gamma(\alpha)} \int_{0}^{t}(t-\tau)^{\alpha-1} f(\tau, u(\tau)) d \tau: f(t, u) \in S_{F}(u)\right\},
$$


where

$$
S_{F}(u)=\left\{f \in L^{1}(J, \mathbb{R}): f(t) \in F(t, u(t)) \text { a.e. } t \in J\right\}
$$

denotes the set of selections of $F$ and $b_{i}: J \rightarrow \mathbb{R}$ is a continuous function such that $B:=\max _{1 \leq i \leq n}\left\{\sup _{t \in J}\left\{\left|b_{i}(t)\right|\right\}\right\}$.

This paper will be organized as follows: In Section 2, we recall briefly some basic definitions and preliminary facts from set-valued analysis which will be used later. In Section 3, we establish the existence of the solution for the single-valued problem

$$
u(t)=\sum_{i=1}^{n} b_{i}(t) u\left(t-\tau_{i}\right)+I^{\alpha} f(t, u(t)) ; 0<\alpha \leq 1, t \in J, f \in F(t, u(t)),
$$

by using the Schauder fixed point theorem, (see [6]) and the uniqueness of the solution by using the Banach fixed point theorem (see [29]). In Section 4, we shall study the existence of solution for the set-valued problem 1.1 when $F$ has convex values, as well when it has with non-convex values, via the single-valued problem as well as fixed point theorems of the set-valued function. In the first case (convex) a fixed point theorem due to Bohnenblust and Karlin (see [8,31]) is used. A fixed point theorem for contraction set-valued functions due to Covitz and Nadler [11] is applied in the second one (non-convex). Also we illustrate our results with examples.

\section{Preliminaries}

In this section, we introduce notations, definitions, and preliminary facts from setvalued analysis which are used throughout this paper. For further background and details pertaining to this section we refer the reader to $[4,7,15,16,19,26,30]$.

$\mathscr{B}:=C[J, \mathbb{R}]$ is the Banach space of all continuous functions from $J$ into $\mathbb{R}$ with the norm

$$
\|u\|=\sup \{|u(t)|: t \in J\}
$$

for each $u \in \mathscr{B} . \mathscr{L}:=L^{1}[J, \mathbb{R}]$ denotes the Banach space of measurable functions $u: J \rightarrow \mathbb{R}$ which are Lebesgue integrable normed by

$$
\|u\|_{L^{1}}=\int_{0}^{T}|u(t)| d t,
$$

for each $u \in \mathscr{L}$. Let $\left(X, \mid\right.$.|) be a normed space, $\mathcal{P}_{c l}(X)=\{Y \in \mathcal{P}(X): Y$ is closed $\}$, $\mathcal{P}_{b}(X)=\{Y \in \mathcal{P}(X): Y$ is bounded $\}, \mathcal{P}_{c p}(X)=\{Y \in \mathcal{P}(X): Y$ is compact $\}$, $\mathcal{P}_{c}(X)=\{Y \in \mathcal{P}(X): Y$ is convex $\}, \mathcal{P}_{c l, c}(X)=\{Y \in \mathcal{P}(X): Y$ is closed and convex $\}, \mathcal{P}_{c p, c}(X)=\{Y \in \mathscr{P}(X): Y$ is compact and convex $\}$. A set-valued function $F: X \rightarrow \mathcal{P}(X)$ is called convex (closed) valued if $F(x)$ is convex (closed) for all $x \in X$. $F$ is called bounded valued on bounded set $B$ if $F(B)=\bigcup_{x \in B} F(x)$ is bounded in $X$ for all $B \in \mathcal{P}_{b}(X)$ i.e. $\sup _{x \in B}\{\sup \{|u|: u \in F(x)\}\}<\infty$. F is called upper semi-continuous (u.s.c) on $X$ if for each $x_{0} \in X$ the set $F\left(x_{0}\right)$ is nonempty closed subset of $X$ and if for each open set $N$ of $X$ containing $F\left(x_{0}\right)$, there exists an open neighborhood $N_{0}$ of $x_{0}$ such that $F\left(N_{0}\right) \subseteq N$.F is u.s.c if 
the set $F^{-1}(A)=\{x \in X: F x \subset A\}$ is open in $X$ for every open set $A$ in $X$. $F$ is called lower semi-continuous (1.s.c) on $X$ if $A$ is any open subset of $X$ then $F^{-1}(A)=\{x \in X: F x \bigcap A \neq \varnothing\}$ is open in $X . F$ is called continuous if it is lower as well as upper semi-continuous on $X . F$ is called compact if for every $M$ bounded subset of $X, F(M)$ is relatively compact. Finally $F$ is called completely continuous if it is upper semi-continuous and compact on $X$.

The following definitions are used in the sequel.

Definition 1. A mapping $p: J \times \mathbb{R} \rightarrow \mathbb{R}$ is said to be Carathéodory if

(i) $t \rightarrow p(t, u)$ is measurable for each $u \in \mathbb{R}$,

(ii) $u \rightarrow p(t, u)$ is continuous a.e. for $t \in J$.

A Carathéodory function $p(t, u)$ is called $L^{1}(J, \mathbb{R})$-Carathéodory if

(iii) for each number $r>0$ there exists a function $h_{r} \in L^{1}(J, \mathbb{R})$ such that $|p(t, u)| \leq$ $h_{r}(t)$ a.e $t \in J$ for all $u \in \mathbb{R}$ with $|u| \leq r$.

A Carathéodory function $p(t, u)$ is called $L_{X}^{1}(J, \mathbb{R})$-Carathéodory if

(iv) there exist a function $h \in L^{1}(J, \mathbb{R})$ such that $|p(t, u)| \leq h(t)$ a.e $t \in J$ for all $u \in \mathbb{R}$ where $h$ is called the bounded function of $p$.

Definition 2. A set-valued function $F: J \rightarrow \mathcal{P}(\mathbb{R})$ is said to be measurable if for any $x \in X$, the function $t \mapsto d(x, F(t))=\inf \{|x-u|: u \in F(t)\}$ is measurable.

Definition 3. A set-valued function $F: J \times \mathbb{R} \rightarrow \mathcal{P}(\mathbb{R})$ is called Carathéodory if

(i) $t \mapsto F(t, x)$ is measurable for each $x \in \mathbb{R}$, and

(ii) $x \mapsto F(t, x)$ is u.s.c. for almost $t \in J$. if

Definition 4. A set-valued function $F: J \times \mathbb{R} \rightarrow \mathcal{P}(\mathbb{R})$ is called $L^{1}$-Carathéodory

(i) $F$ is Carathéodory and

(ii) For each $r>0$, there exists $h_{r} \in L^{1}(J, \mathbb{R})$ such that $\|F(t, u)\|=\sup \{|f|$ : $f \in F(t, u)\} \leq h_{r}(t)$ for all $|u| \leq r$ and for a.e. $t \in J$.

Definition 5 ([12]). A set-valued function $F: J \times \mathbb{R} \rightarrow \mathcal{P}(\mathbb{R})$ is called $\mathbf{L}_{X}^{1}$-Carathéodory if there exists a function $h \in L^{1}(J, \mathbb{R})$ such that

$$
\|F(t, u)\|=\sup \{|f|: f \in F(t, u)\} \leq h(t) \text {, a.e. } t \in J
$$

for all $x \in \mathbb{R}$, and the function $h$ is called a growth function of $F$ on $J \times \mathbb{R}$.

Let $A, B \in \mathcal{P}_{c l}(X)$ and let $a \in A$. Then by

$$
D(a, B)=\inf \{\|a-b\|: b \in B\} \text { and } \rho(A, B)=\sup \{D(a, B): a \in A\}
$$

the function $H: \mathcal{P}_{c l}(X) \times \mathcal{P}_{c l, b}(X) \rightarrow \mathbb{R}^{+}$defined by

$$
H(A, B)=\max \{\rho(A, B), \rho(B, A)\}
$$


is metric and is called Hausdorff metric on $X$. Moreover $\left(\mathcal{P}_{c l, b}(X), H\right)$ is a metric space and $\left(\mathcal{P}_{c l}(X), H\right)$ is a complete metric space(see [21]). It is clear that

$$
H(0, C)=\sup \left\{\|c\|: c \in C ; C \in \mathcal{P}_{b}(X)\right\} .
$$

Definition 6. A set-valued function $F: \mathbb{R} \rightarrow \mathscr{P}_{c l}(\mathbb{R})$ is called

(i) $\gamma$-Lipschitz if and only if there exist $\gamma>0$ such that

$$
H(F(x), F(y)) \leq \gamma\|x-y\|, \text { for each } x, y \in X
$$

the constant $\gamma$ is called a Lipschitz constant.

(ii) a contraction if and only if it is $\gamma$-Lipschitz with $\gamma<1$.

Definition 7. A set-valued function $F: J \times \mathbb{R} \rightarrow \mathcal{P}_{c l}(\mathbb{R})$ is called

(i) $\gamma(t)$-Lipschitz if and only if there exists $\gamma \in L^{1}\left(J, \mathbb{R}^{+}\right)$such that

$$
H(F(t, x), F(t, y)) \leq \gamma(t)\|x-y\|, \text { for each } x, y \in X .
$$

(ii) a contraction if and only if it is $\gamma(t)$-Lipschitz with $\|\gamma\|<1$

The following remark and lemmas are used in the sequel.

Remark 1 ([5]). Let $M \subset X$. If $F: M \rightarrow \mathcal{P}(X)$ is closed and $F(M)$ is relatively compact then $F$ is u.s.c. on $M$. And if $F: X \rightarrow \mathcal{P}(X)$ is closed and compact operator then $F$ is u.s.c. on $X$.

Lemma 1 (Bohnenblust-Karlin). Let $M$ be a closed and convex subset of the Banach space $X$ and $F: M \rightarrow \mathcal{P}(M)$ a set-valued function. Suppose that:

(i) the set $F(M)$ is relatively compact;

(ii) the set-value function $F$ is u.s.c on $M$;

(iii) the set $F(x)$ is nonempty closed and convex for all $x \in M$.

Then there is $x \in M$ such that $x \in F(x)$.

Lemma 2 (Covitz-Nadler). Let $(X, d)$ be a complete metric space. If $G: X \rightarrow$ $\mathcal{P}_{c l}(X)$ is a contraction, then $G$ has a fixed point.

\section{SingLE-VALUED PROBLEM}

In this section we prove that the integral equation 1.2 has a solution $u(t)$ on $J$. Let us formulate the following assumption:

(H1) The function $f$ is $L_{X}^{1}(J, \mathbb{R})$-Carathéodory with bound function $h \in L^{1}(J, \mathbb{R})$ such that $|f(t, u)| \leq h(t)$ a.e. $t \in J$ for all $u \in \mathbb{R}$.

Theorem 1. Let $(H 1)$ hold. If $1-n B>0$ then equation 1.2 has at least one solution $u(t)$ on $J$. 
Proof. Define an operator $P$ by

$$
(P u)(t):=\sum_{i=1}^{n} b_{i}(t) u\left(t-\tau_{i}\right)+I^{\alpha} f(t, u(t)) .
$$

Set $r:=\frac{T^{\alpha}\|h\|}{\Gamma(\alpha+1)(1-n B)}$, then by the assumption of the theorem and the properties of the fractional calculus we obtain that

$$
\begin{aligned}
& |(P u)(t)| \leq \sum_{i=1}^{n}\left|b_{i}(t) \| u\left(t-\tau_{i}\right)\right|+I^{\alpha}|f(t, u(t))| \\
& \quad \leq n B\|u\|+I^{\alpha}|h(t)| \leq n B\|u\|+\|h\| \frac{T^{\alpha}}{\Gamma(\alpha+1)} .
\end{aligned}
$$

So we obtain that

$$
\|P u\| \leq \frac{T^{\alpha}\|h\|}{\Gamma(\alpha+1)(1-n B)} .
$$

That is $P: B_{r} \rightarrow B_{r}$. Then $P$ maps $B_{r}$ into itself. In fact, $P$ maps the convex closure of $P\left[B_{r}\right]$ into itself. Since $f$ is bounded on $B_{r}$, thus $P\left[B_{r}\right]$ is equicontinuous and the Schauder fixed point theorem shows that $P$ has at least one fixed point $u \in \mathscr{B}=$ $C[J, \mathbb{R}]$ such that $P u=u$, which is corresponding to the solution of 1.2.

For the uniqueness let us consider the following assumption

(H2) The function $f$ satisfies that there exists a function $\ell(t) \in L^{1}\left(J, \mathbb{R}^{+}\right)$such that for each $u, v \in C[J, \mathbb{R}]$ we have

$$
|f(t, u)-f(t, v)| \leq \ell(t)\|u-v\| .
$$

Theorem 2. Let (H2) hold. If $\frac{n B \Gamma(\alpha+1)+T^{\alpha}\|\ell\|}{\Gamma(\alpha+1)}<1$, then 1.2 has a unique solution $u(t)$ on $J$.

Proof. Assume the operator $P$ defined in equation 3.1 then we have

$$
\begin{aligned}
|(P u)(t)-(P v)(t)| \leq & \sum_{i=1}^{n}\left|b_{i}(t) \| u\left(t-\tau_{i}\right)-v\left(t-\tau_{i}\right)\right|+I^{\alpha}|f(t, u(t))-f(t, v(t))| \\
& \leq n B\|u-v\|+I^{\alpha} \ell(t)\|u-v\| \\
& \leq n B\|u-v\|+\frac{\|\ell\| T^{\alpha}}{\Gamma(\alpha+1)}\|u-v\| \\
& =\left(n B+\frac{\|\ell\| T^{\alpha}}{\Gamma(\alpha+1)}\right)\|u-v\| .
\end{aligned}
$$


Hence by the assumption of the theorem we have that $P$ is a contraction mapping. Then in view of Banach fixed point Theorem, $P$ has a unique fixed point which is corresponding to the solution of equation 1.2.

\section{SET-VALUED PROBLEM}

In this section we establish the existence results for the integral inclusion 1.1 when the right hand side is convex as well as when it is non-convex valued. The study will be taken in view of the single-valued theorems as well as fixed point theorems of set-valued function. Let us illustrate the following assumption

(H3) The set-valued function $F: J \times \mathbb{R} \rightarrow \mathcal{P}_{c l, c}(\mathbb{R})$ is $L_{X}^{1}(J, \mathbb{R})$-Carathéodory with a growth function $h \in L^{1}(J, \mathbb{R})$ such that $\|F(t, u)\| \leq h(t)$ a.e $t \in J$ for all $u \in \mathbb{R}$.

Theorem 3. Let (H3) hold. If $F$ is lower semi-continuous (l.s.c) and $1-n B>0$ then the integral inclusion 1.1 has at least one solution $u(t)$ on $J$.

Proof. The proof here is depending on the single-valued problem. For each $u(t)$ in $\mathbb{R}$ the set $S_{F}(u)$ is nonempty, since by $(H 3), F$ has a non-empty measurable selection (see [9]). Thus there exists a function $f(t, u) \in F$ where $f$ is a $L_{X}^{1}(J, \mathbb{R})$ Carathéodory function with a bounded function $h \in L^{1}(J, \mathbb{R})$ such that $\|f(t, u)\| \leq$ $h(t)$ a.e $t \in J$ for all $u \in \mathbb{R}$. Hence the assumptions of Theorem 2 are satisfied so the integral inclusion 1.1 has a solution.

Theorem 4. (Convex case) Let (H3) hold. If $1-n B>0$ then the integral inclusion 1.1 has at least one solution $u(t)$ on $J$.

Proof. Now we shall show that the assumptions of Lemma 1 are satisfied. Consider the following space $\Omega:=\{u: J \rightarrow \mathbb{R} \mid u(t) \in C[J, \mathbb{R}]\}$ which is a Banach space endow with the sup. norm. Consider the set-valued operator $N: \Omega \rightarrow \mathcal{P}(\Omega)$ defined by

$$
(N u)(t):=\left\{u \in \Omega: u(t)=\sum_{i=1}^{n} b_{i}(t) u\left(t-\tau_{i}\right)+I^{\alpha} f(t, u(t)) ; f \in S_{F}(u)\right\} .
$$

We shall show that $N$ satisfies the assumptions of Lemma 1. The proof will be given by several steps. Define a set $K:=\left\{u \in \Omega:\|u\| \leq r:=\frac{T^{\alpha}\|h\|}{\Gamma(\alpha+1)(1-n B)}\right\}$ such that $\sup \{|u(t)|: u \in \Omega\}<\infty$. It is clear that $K$ is nonempty, bounded, closed and convex subset of $\Omega$. Note that by (H3) $S_{F}(u) \neq \varnothing$.

Step 1: $N(K) \subset K$. Let $u \in K$, we must show that $N(u) \in K$. For all $h(t) \in N(u)$, we have

$$
|h(t)| \leq \sum_{i=1}^{n}\left|b_{i}(t)\right|\left|u\left(t-\tau_{i}\right)\right|+I^{\alpha}|f(t, u(t))|
$$




$$
\begin{aligned}
\leq n B\|u\|+I^{\alpha}|h(t)| \leq n B\|u\|+\frac{\|h\|_{L^{1}} T^{\alpha}}{\Gamma(\alpha+1)} & \\
& \leq n r B+(1-n B) r=r .
\end{aligned}
$$

Step 2: $N(K)$ is relatively compact set. We must show that $N$ is a compact operator. Since $K$ is bounded closed and convex set and $N(K) \subset K$, it follows that $N(K)$ is bounded closed and convex set. Moreover, for $0 \leq t_{1} \leq t_{2} \leq T$ such that $\left|t_{2}-t_{1}\right|<\delta, \delta>0$ and $u \in K$ then for all $h(t) \in N(u)$,

$$
\begin{gathered}
\left|h\left(t_{1}\right)-h\left(t_{2}\right)\right| \\
=\left|\sum_{i=1}^{n} b_{i}\left(t_{1}\right) u\left(t_{1}-\tau_{i}\right)+I^{\alpha} f\left(t, u\left(t_{1}\right)\right)-\sum_{i=1}^{n} b_{i}\left(t_{2}\right) u\left(t_{2}-\tau_{i}\right)-I^{\alpha} f\left(t, u\left(t_{2}\right)\right)\right| \\
\leq 2 n B\|u\|+\left|I^{\alpha} f\left(t, u\left(t_{1}\right)\right)-I^{\alpha} f\left(t, u\left(t_{2}\right)\right)\right| \\
\leq 2 n B\|u\|+\left[\frac{\|f\|}{\Gamma(\alpha+1)}\right]\left|\left(t_{1}^{\alpha}-t_{2}^{\alpha}+2\left(t_{2}-t_{1}\right)^{\alpha}\right)\right| \\
\leq 2 n B\|u\|+\left[\frac{2\|f\|}{\Gamma(\alpha+1)}\right]\left|\left(t_{2}-t_{1}\right)^{\alpha}\right| \\
\leq 2 n B\|u\|+\left[\frac{2\|f\|}{\Gamma(\alpha+1)}\right]\left|\left(t_{2}-t_{1}\right)\right|^{\alpha} \\
\leq 2\left[n B\|u\|+\frac{2 \delta^{\alpha}\|f\|}{\Gamma(\alpha+1)},\right.
\end{gathered}
$$

which is independent of $u$, and that implies that $N(K)$ is equicontinuous. An application of Arzela-Ascoli Theorem yields that $N$ maps $K$ into a compact set in $\Omega$, that is $N ; K \rightarrow \mathcal{P}(\Omega)$ is a compact operator. Thus $N(K)$ is relatively compact set.

Step 3: $N$ is upper semi-continuous on $K$. Since $N: K \rightarrow K$ is closed and compact and that $N(K)$ is relatively compact set then in view of Remark 1, we obtain that $N$ is u.s.c on $K$.

Step 4: $N(u)$ is a convex. Let $h_{1}(t), h_{2}(t) \in N(u)$ then there exist two functions $f_{1}, f_{2} \in S_{F}(u)$ such that

$$
h_{j}(t)=\sum_{i=1}^{n} b_{i}(t) u\left(t-\tau_{i}\right)+I^{\alpha} f_{j}(t, u(t)) ; j=1,2 .
$$

Let $0 \leq \delta \leq 1$, then for each $t \in J$ we have

$$
\begin{gathered}
{\left[\delta h_{1}+(1-\delta) h_{2}\right](t)=\delta\left[\sum_{i=1}^{n} b_{i}(t) u\left(t-\tau_{i}\right)+I^{\alpha} f_{1}(t, u(t))\right]} \\
+(1-\delta)\left[\sum_{i=1}^{n} b_{i}(t) u\left(t-\tau_{i}\right)+I^{\alpha} f_{2}(t, u(t))\right]
\end{gathered}
$$




$$
\begin{gathered}
=\sum_{i=1}^{n} b_{i}(t)\left[\delta u\left(t-\tau_{i}\right)+(1-\delta) u\left(t-\tau_{i}\right)\right] \\
+\left[\delta I^{\alpha} f_{1}(t, u(t))+(1-\delta) I^{\alpha} f_{2}(t, u(t))\right] \\
=\sum_{i=1}^{n} b_{i}(t)\left[\delta u\left(t-\tau_{i}\right)+(1-\delta) u\left(t-\tau_{i}\right)\right]+I^{\alpha}\left[\delta f_{1}(t, u(t))+(1-\delta) f_{2}(t, u(t))\right] \\
=\sum_{i=1}^{n} b_{i}(t) u\left(t-\tau_{i}\right)+I^{\alpha}\left[\delta f_{1}(t, u(t))+(1-\delta) f_{2}(t, u(t))\right]
\end{gathered}
$$

by the convexity of $S_{F}(u)$, and $F(t, u)$ implies that $\left[\delta h_{1}+(1-\delta) h_{2}\right](t) \in N(u)$. Hence $N(u)$ is convex. As a consequence of Lemma 1, we deduce that $N$ has a fixed point which is a solution for the integral inclusion 1.1. Hence the proof is complete.

In order to study the existence for the problem 1.1 in non-convex case, we introduce the following assumptions.

(H4) $F: J \times \mathbb{R} \rightarrow \mathcal{P}_{c l}(\mathbb{R}),(t,.) \mapsto F(t, u)$ is measurable for each $u \in \mathbb{R}$.

(H5) $F: J \times \mathbb{R} \rightarrow \mathcal{P}_{c l}(\mathbb{R})$ is $\ell(t)$-Lipschitz

$$
H(F(t, u), F(t, v)) \leq \ell(t)\|u-v\| .
$$

Theorem 5 (Non-convex case). Let (H4) and (H5) hold. If

$$
\frac{n B \Gamma(\alpha+1)+T^{\alpha}\|\ell\|_{L^{1}}}{\Gamma(\alpha+1)}<1
$$

then the integral inclusion 1.1 has at least one solution $u(t)$ on $J$.

Proof. For each $u(t)$ in $\mathbb{R}$ the set $S_{F}(u)$ is nonempty since by $(H 4), F$ has a nonempty measurable selection (see [9]). Then there exists a function $f(t, u) \in F$ such that $f$ is $\ell(t)$-Lipschitz. Thus by the assumption of the theorem and by Theorem 2 , the inclusion 1.1 has a solution. Hence the proof is done in view of the singlevalued problem.

In order to study the existence for problem 1.1 by using Lemma 2, we must show that $N: \Omega \rightarrow \mathcal{P}(\Omega)$ defined in 4.1 is a contraction set-valued mapping. The proof will be given in two steps.

Step 1: $N(u) \in \mathcal{P}_{c l}(\Omega)$ for each $u \in \Omega$. Let $\left\{u_{m}\right\}_{m \geq 0} \in N(u)$ such that $u_{m} \rightarrow \widetilde{u}$ in $\Omega$. Then $\widetilde{u} \in \Omega$ and there exists $f_{m} \in S_{F}(u)$ such that for $t \in J$

$$
u_{m}(t)=\sum_{i=1}^{n} b_{i}(t) u\left(t-\tau_{i}\right)+I^{\alpha} f_{m}(t, u(t)) .
$$


Using the fact that $F$ has closed values, we get that $f_{m}$ converges to $f$ in $L^{1}(J, \mathbb{R})$ and hence $f \in S_{F}(u)$. Then for each $t \in J$

$$
u_{m}(t) \rightarrow \widetilde{u}(t)=\sum_{i=1}^{n} b_{i}(t) u\left(t-\tau_{i}\right)+I^{\alpha} f(t, u(t)) .
$$

So $\widetilde{u} \in N(u)$.

Step 2: There exists $\gamma<1$ such that

$$
H(N(u), N(v)) \leq \gamma\|u-v\|_{\Omega}, \text { for each } u, v \in \Omega .
$$

Let $u, v \in \Omega$. Then by (H5) there exists $f \in F$ that satisfies

$$
|f(t, u)-f(t, v)| \leq \ell(t)\|u-v\|_{\Omega} .
$$

Then $h_{1}(t) \in N(u)$ where

$$
h_{1}(t)=\sum_{i=1}^{n} b_{i}(t) u\left(t-\tau_{i}\right)+I^{\alpha} f(t, u(t)) .
$$

Then for $h_{2}(t) \in N(v)$, where

$$
h_{2}(t)=\sum_{i=1}^{n} b_{i}(t) v\left(t-\tau_{i}\right)+I^{\alpha} f(t, v(t)),
$$

we have

$$
\begin{gathered}
\left|h_{1}(t)-h_{2}(t)\right| \leq \sum_{i=1}^{n} b_{i}(t)\left|u\left(t-\tau_{i}\right)-v\left(t-\tau_{i}\right)\right|+\left|I^{\alpha} f(t, u(t))-I^{\alpha} f(t, v(t))\right| \\
\leq \sum_{i=1}^{n} b_{i}(t)\left|u\left(t-\tau_{i}\right)-v\left(t-\tau_{i}\right)\right|+I^{\alpha}|f(t, u(t))-f(t, v(t))| \\
\leq n B\|u-v\|_{\Omega}+\frac{T^{\alpha}}{\Gamma(\alpha+1)}|f(t, u(t))-f(t, v(t))| \\
\leq n B\|u-v\|_{\Omega}+\frac{T^{\alpha}}{\Gamma(\alpha+1)} \ell(t)\|u-v\|_{\Omega} \\
\leq n B\|u-v\|_{\Omega}+\frac{T^{\alpha}\|\ell\|_{L^{1}}}{\Gamma(\alpha+1)}\|u-v\|_{\Omega} \\
=\left[n B+\frac{T^{\alpha}\|\ell\|_{L^{1}}}{\Gamma(\alpha+1)}\right]\|u-v\|_{\Omega} .
\end{gathered}
$$

Denote

$$
\gamma:=\left[\frac{n B \Gamma(\alpha+1)+T^{\alpha}\|\ell\|_{L^{1}}}{\Gamma(\alpha+1)}\right]
$$

It follows that

$$
H(N(u), N(v)) \leq \gamma\|u-v\|_{\Omega}, \text { for each } u, v \in \Omega,
$$


where $\gamma<1$. It follows that $N$ is a contraction set-valued mapping. Then, in view of Lemma 2, $N$ has a fixed point which is corresponding to a solution of inclusion 1.1. This completes the proof.

Example 1. Let $J=[0,1]$ denote a closed and bounded interval in $\mathbb{R}$. Consider the problem

$$
u(t) \in \frac{t^{3}}{8} u\left(t-\tau_{1}\right)+\frac{t^{4}}{12} u\left(t-\tau_{2}\right)+\frac{1}{4} u\left(t-\tau_{3}\right)+I^{\alpha} F(t, u) ; t \in[0,1]
$$

where $n=3, \alpha=0.5, B=0.25$ and

$$
F(t, u)=\left\{\begin{array}{l}
h(t), \text { if } u<0 ; \\
{\left[h(t) \exp \frac{-u(t)}{2}, h(t)\right], \text { if } u \geq 0 .}
\end{array}\right.
$$

It is clear that $F$ is $L_{X}^{1}(J, \mathbb{R})$-Carathéodory with a growth function $h \in L^{1}\left(J, \mathbb{R}^{+}\right)$ such that $\|F(t, u)\| \leq h(t)$ a.e $t \in J$ for all $u \in \mathbb{R}$. Therefore if $\|h\| \leq \frac{\sqrt{\pi}}{8}$ then in view of Theorem 4, the problem 4.2 has a solution.

Example 2. Let $J=[0,1]$ denote a closed and bounded interval in $\mathbb{R}$. Consider the problem

$$
u(t) \in \frac{t}{5} u\left(t-\tau_{1}\right)+\frac{t^{2}}{10} u\left(t-\tau_{2}\right)+I^{\alpha} F(t, u) ; t \in[0,1]
$$

where $n=2, \alpha=0.5, B=\frac{1}{5}$ and

$$
F(t, u)=\left\{\begin{array}{cr}
0 & \text { if } u<0 \\
{\left[0, \frac{u(t)}{4}\right]} & \text { if } u \geq 0 .
\end{array}\right.
$$

It is clear that $F$ is $\ell(t)$-Lipschitzian set-valued function with $\|\ell\|=\frac{1}{4}$. Therefore, for $\gamma=0.6<1$, we have that $F$ is a contraction set-valued mapping. Thus, in view of Theorem 5, the problem 4.3 has a solution.

\section{REFERENCES}

[1] N. U. Ahmed and X. Xiang, "Existence of solutions for a class of nonlinear evolution equations with nonmonotone perturbations." Nonlinear Anal., vol. 22, no. 1, pp. 81-89, 1994.

[2] N. U. Ahmed and K. L. Teo, Optimal control of distributed parameter systems. New YorkAmsterdam: North-Holland Publishing Co., 1981.

[3] S. Aizicovici and V. Staicu, "Continuous selections of solution sets to volterra integral inclusions in banach spaces,” Electron. J. Differential Equations, vol. 2006, no. 1, pp. 1-11, 2006.

[4] J.-P. Aubin and A. Cellina, Differential inclusions. Set-valued maps and viability theory, ser. Fundamental Principles of Mathematical Sciences. Berlin: Springer-Verlag, 1984, vol. 264.

[5] C. Avramescu, "A fixed point theorem for multivalued mappings," Electron. J. Qual. Theory Differ. Equ., no. 17, pp. 1-10, 2004.

[6] K. Balachandar and J. Dauer, Elements of control theory. Narosa Publishing House, 1999.

[7] V. Barbu, Nonlinear semigroups and differential equations in Banach spaces. Leiden: Noordhoff International Publishing, 1976. 
[8] H. F. Bohnenblust and S. Karlin, On a theorem of Ville, ser. Annals of Mathematics Studies. Princeton: Princeton University Press, 1950, vol. 24.

[9] C. Castaing and M. Valadier, Convex analysis and measurable multifunctions, ser. Lecture Notes in Mathematics. Berlin-New York: Springer-Verlag, 1977, vol. 580.

[10] A. Cernea, "Existence for nonconvex integral inclusions via fixed points," Arch. Math. (Brno), vol. 39, no. 4, pp. 293-298, 2003.

[11] K. Deimling, Multivalued differential equations, ser. de Gruyter Series in Nonlinear Analysis and Applications. Berlin: Walter de Gruyter \& Co., 1992, vol. 1.

[12] B. C. Dhage, "Multi-valued operators and fixed point theorems in banach algebras. i." Taiwanese J. Math., vol. 10, no. 4, pp. 1025-1045, 2006.

[13] A. M. A. El-Sayed and A. G. Ibrahim, "Multivalued fractional differential equations," Appl. Math. Comput., vol. 68, no. 1, pp. 15-25, 1995.

[14] A. M. A. El-Sayed and A. G. Ibrahim, "Set-valued integral equations of fractional-orders," Appl. Math. Comput., vol. 118, no. 1, pp. 113-121, 2001.

[15] S. Hu and N. S. Papageorgiou, Handbook of multivalued analysis. Vol. I., ser. Mathematics and its Applications. Dordrecht: Kluwer Academic Publishers, 1997, vol. 419.

[16] S. Hu and N. S. Papageorgiou, Handbook of multivalued analysis. Vol. II., ser. Mathematics and its Applications. Dordrecht: Kluwer Academic Publishers, 2000, vol. 500.

[17] A. G. Ibrahim and A. M. A. El-Sayed, "Definite integral of fractional order for set-valued functions," J. Fract. Calc., vol. 11, pp. 81-87, 1997.

[18] M. Kanakaraj and K. Balachandran, "Existence of solutions of sobolev-type semilinear mixed integrodifferential inclusions in banach spaces," J. Appl. Math. Stochastic Anal., vol. 16, no. 2, pp. $163-170,2003$

[19] A. G. Kartsatos and K.-Y. Shin, "Solvability of functional evolutions via compactness methods in general banach spaces," Nonlinear Anal., vol. 21, no. 7, pp. 517-535, 1993.

[20] V. Kiryakova, Generalized fractional calculus and applications, ser. Pitman Research Notes in Mathematics Series. New York: Longman Scientific \& Technical, Harlow; copublished in the United States with John Wiley \& Sons, Inc., 1994, vol. 301.

[21] M. Kisielewicz, Differential inclusions and optimal control, ser. Mathematics and its Applications (East European Series). Dordrecht: Kluwer Academic Publishers Group, 1991, vol. 44.

[22] K. S. Miller and B. Ross, An introduction to the fractional calculus and fractional differential equations, ser. A Wiley-Interscience Publication. New York: John Wiley \& Sons, Inc., 1993

[23] K. B. Oldham and J. Spanier, The fractional calculus, ser. Mathematics in Science and Engineering. New York-London: Academic Press, 1974, vol. 111.

[24] N. S. Papageorgiou, "On integral inclusions of volterra type in banach spaces," Czechoslovak Math. J., vol. 42(117), no. 4, pp. 693-714, 1992.

[25] N. S. Papageorgiou, "On nonconvex valued volterra integral inclusions in banach spaces," Czechoslovak Math. J., vol. 44(119), no. 4, pp. 631-648, 1994.

[26] N. H. Pavel, Nonlinear evolution operators and semigroups, ser. Lecture Notes in Mathematics. Berlin: Springer-Verlag, 1987, vol. 1260.

[27] I. Podlubny, Fractional differential equations, ser. Mathematics in Science and Engineering. San Diego: Academic Press, Inc., 1999, vol. 198.

[28] S. G. Samko, A. A. Kilbas, and O. I. Marichev, Fractional integrals and derivatives. Yverdon: Gordon and Breach Science Publishers, 1993.

[29] D. R. Smart, Existence for nonconvex integral inclusions via fixed points, ser. Fixed PointTheorems. Cambridge: Cambridge University Press, 1980.

[30] I. I. Vrabie, Compactness methods for nonlinear evolutions, ser. Pitman Monographs and Surveys in Pure and Applied Mathematics. New York: Longman Scientific \& Technical, Harlow; John Wiley \& Sons, Inc., 1987, vol. 32. 
[31] E. Zeidler, Nonlinear functional analysis and its applications. I., ser. Fixed-point theorems. New York: Springer-Verlag, 1986.

\section{Authors' addresses}

Rabha W. Ibrahim

School of Mathematical Sciences, Faculty of science and Technology University Kebangsaan Malaysia, Bangi 43600, Selangor Darul Ehsan, Malaysia

E-mail address: rabhaibrahim@yahoo.com

\section{Hamid A. Jalab}

Department of Computer System and Technology, Faculty of Computer Science and Information Technology, University Malaya, 50603 Kuala Lumpur, Malaysia

E-mail address: hamidjalab@um.edu.my 